\title{
Ambient temperature and genotype differentially affect developmental and phenotypic plasticity in Arabidopsis thaliana
}

\author{
Carla Ibañez ${ }^{1,2 \dagger}$, Yvonne Poeschl ${ }^{3,4 \dagger}$, Tom Peterson², Julia Bellstädt ${ }^{1,2}$, Kathrin Denk ${ }^{1,2}$, Andreas Gogol-Döring ${ }^{3,4}$, \\ Marcel Quint ${ }^{1,2}$ and Carolin Delker ${ }^{1,2^{*}}$ (D)
}

\begin{abstract}
Background: Global increase in ambient temperatures constitute a significant challenge to wild and cultivated plant species. Forward genetic analyses of individual temperature-responsive traits have resulted in the identification of several signaling and response components. However, a comprehensive knowledge about temperature sensitivity of different developmental stages and the contribution of natural variation is still scarce and fragmented at best.

Results: Here, we systematically analyze thermomorphogenesis throughout a complete life cycle in ten natural Arabidopsis thaliana accessions grown under long day conditions in four different temperatures ranging from 16 to $28^{\circ} \mathrm{C}$. We used $\mathrm{Q}_{10}, \mathrm{GxE}$, phenotypic divergence and correlation analyses to assess temperature sensitivity and genotype effects of more than 30 morphometric and developmental traits representing five phenotype classes. We found that genotype and temperature differentially affected plant growth and development with variing strengths. Furthermore, overall correlations among phenotypic temperature responses was relatively low which seems to be caused by differential capacities for temperature adaptations of individual accessions.
\end{abstract}

Conclusion: Genotype-specific temperature responses may be attractive targets for future forward genetic approaches and accession-specific thermomorphogenesis maps may aid the assessment of functional relevance of known and novel regulatory components.

Keywords: Arabidopsis, Natural variation, Phenotypic plasticity, Thermomorphogenesis, Phenotyping

\section{Background}

Recurrent changes in ambient temperature provide plants with essential information about time of day and seasons. Yet, even small changes in mean ambient temperatures can profoundly affect plant growth and development resulting in thermomorphogenic changes of plant architecture [1]. In crops like rice, a season-specific increase in the mean minimum temperature of $1{ }^{\circ} \mathrm{C}$ results in a $\sim 10 \%$ reduction in grain yield [2]. Likewise, up to $10 \%$ of the yield stagnation of wheat and barley in Europe over the past two decades can be attributed to climate change [3]. Current projections

\footnotetext{
* Correspondence: carolin.delker@landw.uni-halle.de

${ }^{\dagger}$ Equal contributors

${ }^{1}$ Institute of Agricultural and Nutritional Sciences, Martin Luther University

Halle-Wittenberg, Betty-Heimann-Str. 5, 06120 Halle (Saale), Germany

${ }^{2}$ Department of Molecular Signal Processing, Leibniz Institute of Plant

Biochemistry, Weinberg 3, 06120 Halle (Saale), Germany

Full list of author information is available at the end of the article
}

indicate that mean global air temperatures will increase up to $4.8{ }^{\circ} \mathrm{C}$ by the end of the century $[4,5]$. Global warming will thus have significant implications on biodiversity and future food security.

Elevated ambient temperatures affect of course also wild species in their natural habitats. Long-term phenology studies of diverse plant populations have revealed an advance in first and peak flowering and alterations in the total length of flowering times [6, 7]. Furthermore, estimates project that temperature effects alone will account for the extinction of up to one-third of all European plant species [8]. As the impact of changes in ambient temperature on crop plants and natural habitats emerge, a comprehensive understanding of temperaturemediated growth responses throughout development becomes paramount. 
Our present knowledge on molecular responses to ambient temperature changes has significantly progressed by studies in Arabidopsis thaliana. Model thermomorphogenesis phenotypes such as hypocotyl elongation [9], hyponastic leaf movement [10], and alterations in flowering time have served in various genetic approaches to identify relevant molecular players (reviewed in [1]). In this regard, exploiting naturally occurring genetic variation in these model traits has served as a valuable tool [11-16]. Primary signaling genes/proteins seem to function in response to both temperature and light stimuli. Prominent members of this network are photoreceptors such as CRYPTOCHROME 1 (CRY1 [17]), CRY2 [18] or the recently identified thermosensor PHYTOCHROME B (phyB $[19,20]$ ). Further components include PHYTOCHROME INTERACTING FACTOR 4 (PIF4, ] [21-23], DE-ETIOLATED 1, CONSTITUTIVELY PHOTOMORPHOGENIC 1, ELONGATED-HYPOCOTYL 5 [24-26] and EARLY FLOWERING 3 (ELF3); the latter as a component of the circadian clock $[12,13]$.

The investigation of signaling pathways that translate temperature stimuli into qualitative and quantitative developmental responses has so far largely been limited to either seedling development or flowering time. However, it seems likely that temperature responses in different phases of development either require variations of a canonical signaling pathway or involve at least partially specific signaling components. To enable the dissection of thermomorphogenic signaling at different developmental stages, it is vital to gather a comprehensive understanding of the diversity of temperature reactions throughout plant development.

According to basic principles of thermodynamics, temperature-induced changes in free energy will affect the rates of biochemical reactions. As these effects should occur generally, albeit to different magnitudes, non-selective phenotypic responses can be expected to occur robustly and rather independently of genetic variation. Such traits may therefore be indicative of passive, thermodynamic effects on a multitude of processes. Alternatively, robust temperature responses may be due to thermodynamic effects on highly conserved signaling elements. These may be attractive targets for classic mutagenesis screens to identify the relevant regulatory components. In contrast, natural variation in thermomorphogenesis traits is likely the consequence of variability in one or several specific signaling or response components. It may be addressed by quantitative genetic approaches to identify regulators that contribute to variable temperature responses. Such genes may represent attractive candidates for targeted breeding approaches.

In this study we aim to (i) provide a map of developmental phenotypes that are sensitive to ambient temperature effects throughout a life cycle in the model organism $A$. thaliana, (ii) identify traits that are robustly affected by temperature with little variation among different accessions, and ask (iii) which traits are affected differentially by different genotypes and thus show natural variation in temperature responses.

To realize this, we performed a profiling of numerous developmental and morphological traits which can be sorted into five main categories: juvenile vegetative stage, adult vegetative stage, reproductive stage, morphometric parameters and yield-associated traits. Phenotypes were analyzed in a subset of ten $A$. thaliana accessions which were grown at $16,20,24$, and $28{ }^{\circ} \mathrm{C}$ in climate-controlled environments under long day photoperiods (16 h light/ $8 \mathrm{~h}$ dark). Knowing that even a small randomly selected set of $A$. thaliana accessions covers a wide spectrum of genetic diversity [27], we chose to analyze commonly used lab accessions such as Col-0 and Ws- 2 , accessions known to react hypersensitively to elevated temperature (e.g., Rrs-7, [25, 28]), and parental lines of available mapping populations such as Bay-0, Sha, Lerand Cvi-0 which have been used in previous studies of natural variation in individual thermomorphogenesis traits $[16,18]$.

In addition to a meta-analysis of the phenotypic data, we provide accession-specific developmental reference maps of temperature responses that can serve as resources for future experimental approaches in the analysis of ambient temperature responses in $A$. thaliana.

\section{Methods}

Plant material and growth conditions

Phenotypic parameters (Fig. 1) were assessed in A. thaliana accessions that were obtained from the Nottingham Arabidopsis Stock Centre [29]. Morphological markers and time points of analyses are described in Additional file 1. Sample sizes for each accession-temperature-trait combination and detailed information on stock numbers and geographic origin of accessions are listed in Additional file 2. For seedling stage analyses, surface-sterilized seeds were stratified for 3 days in deionized water at $4{ }^{\circ} \mathrm{C}$ and subsequently placed on $A$. thaliana solution (ATS) nutrient medium [30]. Seeds were germinated and cultivated in climate-controlled growth cabinets (Percival, AR66-L2) at constant temperatures of $16,20,24$ or $28{ }^{\circ} \mathrm{C}$ under long day photoperiods ( $16 \mathrm{~h}$ light $/ 8 \mathrm{~h}$ dark) and a photosynthetically active fluence rate (PAR) of $90 \mu \mathrm{mol} \cdot \mathrm{m}^{-2} \cdot \mathrm{sec}^{-1}$ of cool white fluorescent lamps. We refrained from including a vernalization step because the primary focus of this study was to record morphology and development in response to different constant ambient temperature conditions.

Germination rates were assessed daily on seeds cultivated on horizontal plates. Hypocotyl, root length, and petiole angles were measured in 7 days old seedlings 


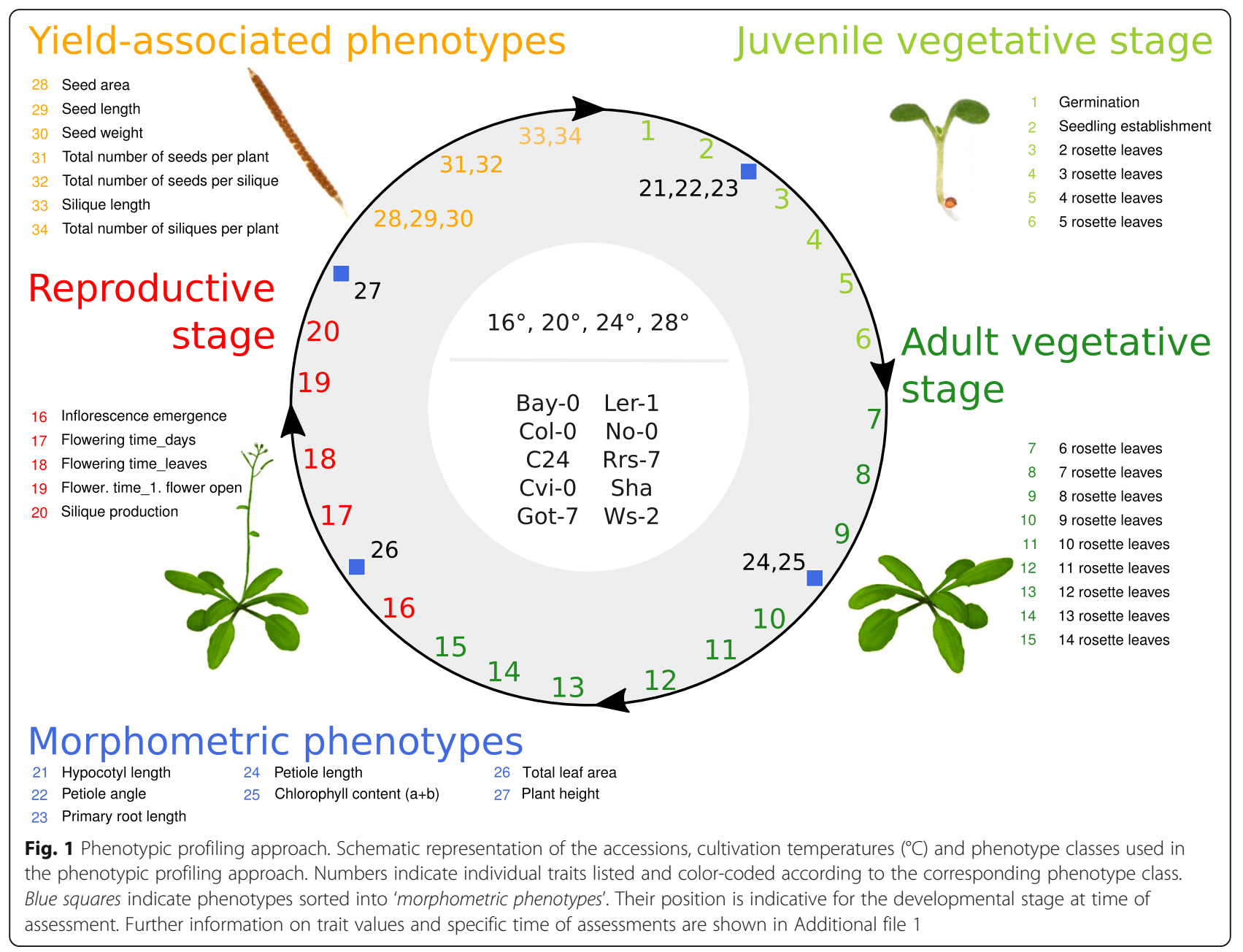

grown on vertically oriented plates using ImageJ [31] and Root Detection [32].

All other analyses were performed on soil-grown plants cultivated in growth cabinets (Percival) at a PAR of $140(+/-20) \mu \mathrm{mol} \cdot \mathrm{m}^{-2} \cdot \mathrm{sec}^{-1}$ and long day photoperiods ( $16 \mathrm{~h}$ light $/ 8 \mathrm{~h}$ dark). After imbibition for 3 days at $4{ }^{\circ} \mathrm{C}$, seeds were grown in individual $5 \times 5 \mathrm{~cm}$ pots, which were randomized twice a week to minimize position effects. Randomization was performed in blocks of 60 pots alternating between 8 positions in the cultivation chamber. In addition, pots within the trays of 60 plants were randomized in groups of 10 twice a week on alternate days to the block randomization of trays. Relative humidity of growth cabinets was maintained at $70 \%$ and plants were watered by subirrigation. Plants $(n>15)$ were photographed daily for subsequent manual determination of phenotypic parameters (leaf number, total leaf area, and petiole length) using Image J [31]. "Total leaf area" was determined in the sense of foliar surface coverage so that overlapping leaf areas were only counted once. Determination of developmental progression largely followed the stages defined in Boyes et al.
[33]. The vegetative growth period was divided in a juvenile phase (germination to initiation of the fifth rosette leave) and an adult vegetative stage (initiation of the sixth rosette leave to floral transition). At transition to the reproductive growth phase, the number of leaves was determined by manual counting in addition to recording the number of days after germination.

Spectrophotometric determination of chlorophyll content was performed as described in [34].

\section{Data analysis}

Visualization and statistical analyses of the data were performed using the software R [35]. Box plots were generated using the boxplot function contained in the graphics package. Heat maps were generated using the heatmap. 2 function contained in the gplots package.

ANOVAs for a single factor (either accession or temperature) and Tukey's 'Honest Significant Difference' test as post hoc test were performed using the anova and TukeyHSD function, respectively, which are both contained in the $\mathrm{R}$ stats package. 
Variation in phenotype expression was analyzed by 2-way ANOVA according to Nicotra [36] and Whitman and Agrawal [37] to test each phenotype for a significant effect of genotype ( $G$, accession) or environment ( $E$, temperature), and a significant genotype by environment interaction (GxE). Reaction norms for each analysis are shown in Additional file 3.

\section{$\mathbf{Q}_{10}$ temperature coefficient}

The $\mathrm{Q}_{10}$ temperature coefficient was calculated according to Loveys [38].

$$
Q_{10}=\left(\frac{P_{w}}{P_{c}}\right)^{\frac{10}{T_{w}-T_{c}}}
$$

where $\mathrm{P}_{\mathrm{w}}$ and $\mathrm{P}_{\mathrm{c}}$ are the trait values at the warmer and cooler temperatures, respectively. $T_{w}$ and $T_{c}$ represent the corresponding temperatures in ${ }^{\circ} \mathrm{C}$. We computed the geometric mean of the six $\mathrm{Q}_{10}$ values of all pairwise temperature combinations for each phenotypic trait to avoid artifacts caused by differential reaction norms/response shapes.

\section{Index of phenotypic divergence $\left(\mathrm{P}_{\mathrm{st}}\right)$}

Calculation of the index of phenotypic divergence $\left(\mathrm{P}_{\text {st }}[39,40]\right)$ as a measure to quantify variation in each phenotypic trait was calculated as previously described by Storz [39] as

$$
P_{\mathrm{st}}=\frac{\sigma_{b}^{2}}{\sigma_{b}^{2}+2 \sigma_{w}^{2}}
$$

where $\sigma_{b}^{2}$ is the variance between populations, and $\sigma_{w}^{2}$ is the variance within populations. The ANOVA framework was used to partition the variances to get unbiased estimates for $\sigma_{b}^{2}$ and $\sigma_{w}^{2}$.

Using the two factorial design, two types of indices of phenotypic variation of a trait/phenotype were considered separately. The index of phenotypic divergence for genotypes $\left(P_{\mathrm{st}}^{\mathrm{gen}}\right)$ at a defined temperature level can be computed to measure the effect/impact of the genotype on the variation whereas the index of phenotypic divergence for temperatures $\left(P_{\mathrm{st}}^{\mathrm{temp}}\right)$ provides a measure for the effect of temperature on the observed variation for individual genotypes.

\section{Principal component analysis (PCA)}

Arithmetic means for each genotype-temperature pair were computed except for six traits (germination, 13 rosette leaves, 14 rosette leaves, silique production, chlorophyl content $(\mathrm{a}+\mathrm{b})$, and total leaf area) due to too many missing values. The remaining 28 traits contained at most eight missing values (randomly distributed).which were replaced per trait by the arithmetic mean of the respective trait values. PCA was perfomed using the prcomp function contained in the $\mathrm{R}$ stats package. Due to the different units and scales of the traits the data was not only set to centered but also to scaled by prcomp.

\section{Pairwise correlation analysis of traits}

Trait values for rosette leave traits were summarized by arithmetic means to trait groups labeled Juvenile vegetative stage (2-5 rosette leaves) and Adult vegetative stage (6-14 rosette leaves), respectively. Similarly, Inflorescence emergence, Flowering time_days and Flower.time_1. flower open were combined to form the trait group Flowering time (days). Spearman correlation coefficients were computed using the $\mathrm{R}$ stats package. Additionally, $p$ values for each Spearman correlation coefficient were computed using the cor.test function. $P$ values were subsequently corrected for multiple testing using the Benjamini-Hochberg correction implemented in the multtest package.

\section{Results}

To assess phenotypic plasticity in a range of ambient temperatures, A. thaliana plants were cultivated in parallel throughout an entire life cycle at four different temperatures $\left(16,20,24\right.$ and $\left.28{ }^{\circ} \mathrm{C}\right)$ under otherwise similar growth conditions (see Methods for further details). More than 30 morphological and developmental traits were recorded representing the following five phenotype classes: juvenile vegetative, adult vegetative, and reproductive stages as well as morphometric and yieldassociated phenotypes (Fig. 1 and Additional file 1).

\section{Temperature responses in the $A$. thaliana reference accession Col-0}

We first focused on Col- 0 as it is a commonly used accession and serves as a point of reference for numerous genetic and physiological analyses. In Col-0, almost all phenotypes analyzed in this study were affected by the cultivation in different ambient temperatures. Only seed weight and maximum height remained constant regardless of the growth temperature (Fig. 2a, Additional file 4). Among the temperature-sensitive traits were several growth-associated phenotypes in the juvenile vegetative stage. Primary root length, hypocotyl and petiole elongation all increased with elevated temperatures which concurs with previously published data $[9,10]$. As another example, yield-related traits, such as the number of siliques per plant and the number of seeds per silique decreased with an increase in ambient temperature (Fig. 2a).

As reported previously, Col-0 plants showed a decrease in developmental time until flowering with increasing ambient temperatures [11]. The transition from the vegetative to the reproductive phase at $28{ }^{\circ} \mathrm{C}$ 


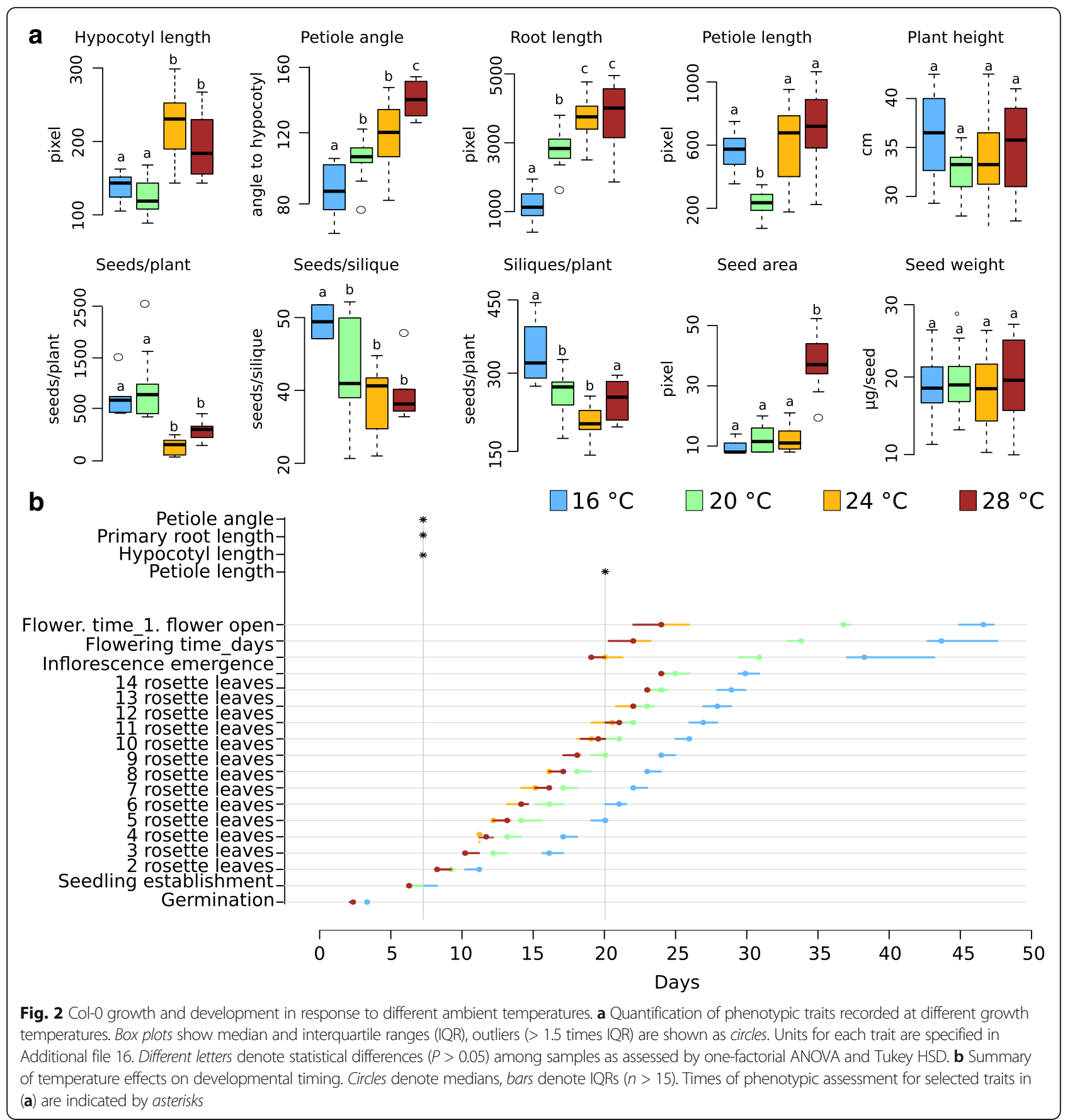

occurred about 25 days earlier than at $16{ }^{\circ} \mathrm{C}$ (Fig. 2a). Similarly, the number of rosette leaves developed at time of bolting differed by approximately 26 leaves between $28{ }^{\circ} \mathrm{C}$ and $16{ }^{\circ} \mathrm{C}$ (Additional file 4b).

The observation that only a very limited number of phenotypes were insensitive to cultivation in different temperatures clearly illustrates the fundamental impact of ambient temperature on plant growth and development.

\section{Natural variation of temperature responses}

To assess whether the observed temperature responses in Col-0 are robust among A. thaliana accessions or which of the responses may be affected by natural variation, phenotypic profiling was performed in nine additional $A$. thaliana accessions parallel to the analysis in Col-0 (Additional file 4, Additional file 5, Additional file 6, Additional file 7, Additional file 8, Additional file 9, 
Additional file 10, Additional file 11, Additional file 12 and Additional file 13). Naturally, a panel of ten accessions does not comprehensively represent the world-wide gene pool of $A$. thaliana. However, it can be expected that even 10 randomly chosen natural accessions represent $\sim 70 \%$ of the allelic diversity in the A. thaliana gene pool [27]. Hence, the general assessment of thermo-responsive development in $A$. thaliana as well as the identification and discrimination between traits that generally seem to exhibit natural variation and those that may be genetically fixed within the gene pool is a realistic aim even with a set of 10 selected accessions.

To approximate and to compare temperature sensitivity of traits among different accessions, we calculated $\mathrm{Q}_{10}$ values for each individual trait and phenotype class for each analyzed genotype [38]. The $\mathrm{Q}_{10}$ quotient represents the factor by which a trait value changes if the ambient temperature increases by $10{ }^{\circ} \mathrm{C}$. We calculated geometric means of all possible pairwise combinations of temperatures to minimize effects potentially caused by different response curves and used the $\log _{2} \mathrm{Q}_{10}$ for visualization as to retain high resolution in the presentation of the data.

Similarly to the response observed in Col-0 (Fig. 2), all analyzed genotypes showed a temperature-induced acceleration of vegetative development as indicated by negative $\log _{2} \mathrm{Q}_{10}$ values with low variability among accessions (Fig. 3a, b, Additional file 4, Additional file 5, Additional file 6, Additional file 7, Additional file 8, Additional file 9, Additional file 10, Additional file 11, Additional file 12 and Additional file 13). Considerably higher variation was observed in $\log _{2} \mathrm{Q}_{10}$ values of traits related to reproductive stages. As all accessions investigated were principally able to flower despite the lack of an extended cold period, none of them strictly required a vernalization treatment to transition to the reproductive phase. In contrast to the other accessions, Got-7 and Rrs-7, however, showed a significant delay in flowering time with increasing temperature (Fig. 3b). Got-7, for example, did not flower within the first 85 days of cultivation when grown in 24 or $28{ }^{\circ} \mathrm{C}$. Thus, initiated leaf senescence at bolting stage prevented accurate determination of leaf number at the onset of flowering.

A direct comparison of leaf number and time of development further corroborates a sudden increase in the overall variation among accessions at the transition to flowering (Additional file 14). However, at $16{ }^{\circ} \mathrm{C}$ and $20{ }^{\circ} \mathrm{C}$ several accessions contribute to the overall variability in the graph, whereas at $24{ }^{\circ} \mathrm{C}$ and $28{ }^{\circ} \mathrm{C}, \mathrm{C} 24$ and Rrs-7 are the main determinants of variation due to their massive number of leaves corresponding to an extension of the vegetative growth phase (Additional file 14). Got-7 likely would increase the interspecific variation in flowering time at 24 and $28{ }^{\circ} \mathrm{C}$, but is missing in this representation due to the lack of flowering transition within 85 days.
Here, the lack of vernalization may at least partially be a significant factor because cold treatment is explicitly recommended to induce earlier flowering for several Got-7 lines available at NASC/ABRC [41]. Natural variation in vernalization regulators may contribute to this phenotype. However, as all accessions were able to flower at temperatures of 16 and $20^{\circ} \mathrm{C}$ vernalization does not seem to be an essential requirement.

Taken together, juvenile and adult vegetative development responded highly conserved, whereas the reproductive stage and yield-associated traits showed higher variation between accessions and within individual accession, as indicated by the ranges/dimensions of the box plots in Fig. 3a. Here, $\log _{2}\left(\mathrm{Q}_{10}\right)$ values of individual traits were summarized by phenotype classes. Thus, high variation within a phenotype class indicates that temperature effects on individual traits within that class are highly variable. The strongest variation within individual accessions was observed for morphometric phenotypes as represented by a wide whisker range and large boxes for all analyzed accessions. This is indicative for a high degree of diversity in the temperature responses of different morphometric traits. In contrast, a high variation of $\log _{2}\left(\mathrm{Q}_{10}\right)$ values between different accessions implicates differential responses of different genotypes which was most prominent in reproductive stage traits.

The differential variances of $\log _{2} \mathrm{Q}_{10}$ values among the two vegetative and the other phenotype classes indicated that genotype and environment effects may contribute differentially to phenotypic plasticity of different traits. We first used a 2-factorial ANOVA to assess which phenotypes show significant changes that can be attributed to genotype ( $\mathrm{G}$, accession), environment (E, temperature), and/or GxE interaction. Subsequently, we used the variance partitioning approach $[39,40,42,43]$ to dissect and quantify the extent of the individual genotype and temperature effects on the phenotypic variation in more detail.

\section{Genotype, environment, and GxE interaction analysis}

Each phenotypic trait was subjected to a 2-factorial ANOVA to address which of the analyzed factors (G, E, $\mathrm{GxE}$ ) had significant effects on the trait. Reaction norm plots for each phenotype are shown in Additional file 3. Each of the analyzed traits showed significant effects of genotype, environment (temperature) and $\mathrm{GxE}$ interaction (Additional file 15). Surprisingly, this included all juvenile and adult vegetative stages despite their seemingly uniform impression of temperature responses given by the $\mathrm{Q}_{10}$ values (Fig. 3a, b).

To assess genotype and temperature contributions in a more quantitative manner, we next used a variance partitioning approach [39, 40, 42, 43]. Specifically, we calculated the index of phenotypic divergence $\left(\mathrm{P}_{\mathrm{st}},[39]\right)$ 


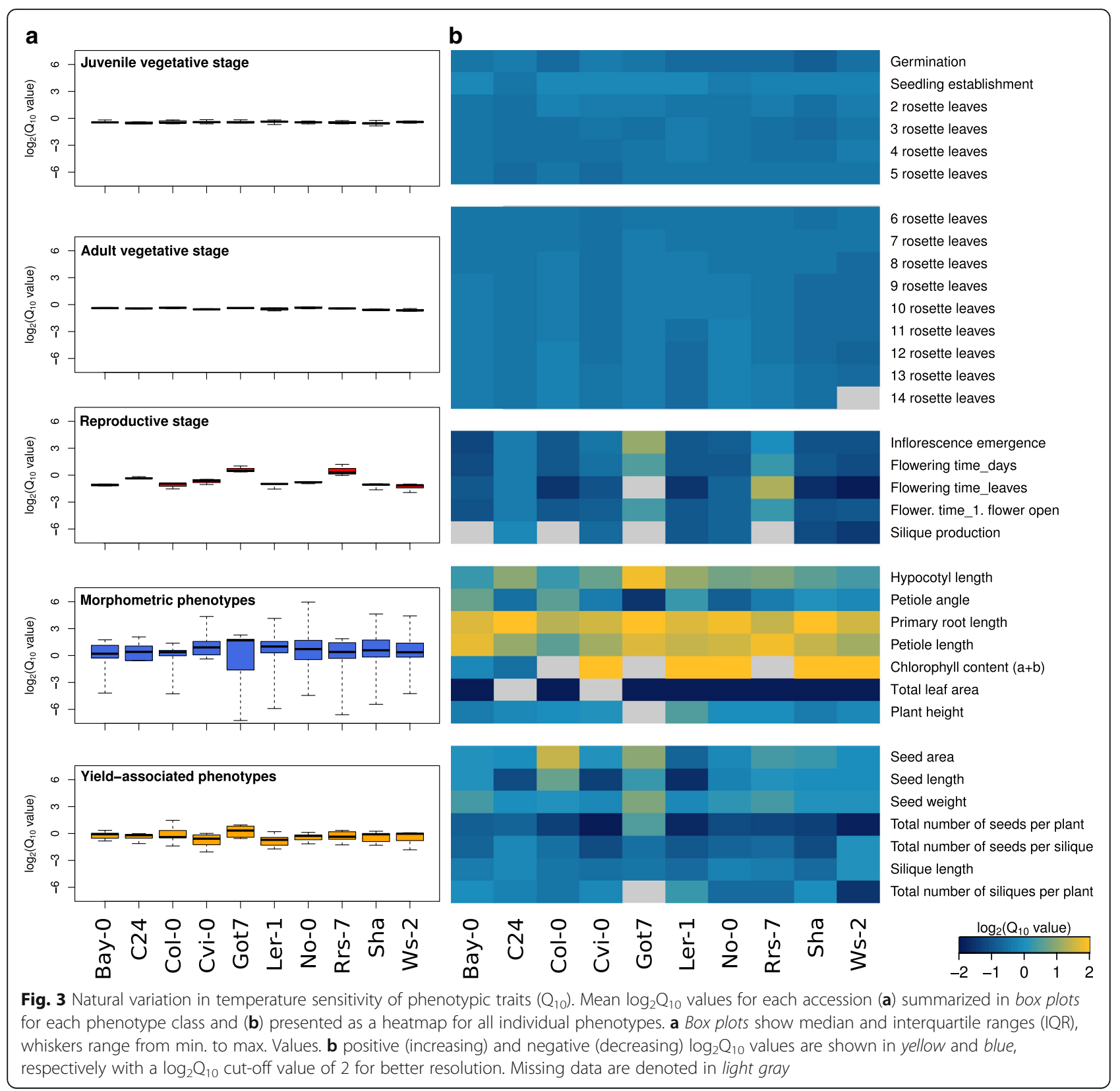

at each analyzed temperature as a measure of genotype effects $P_{\mathrm{st}}^{\mathrm{gen}}$ on the trait of interest (Additional file 16a). To complement this analysis, we also estimated the variation occurring across temperatures $P_{\mathrm{st}}^{\text {temp }}$ for each of the analyzed accessions (Additional file 16b), which enabled us to assess the temperature effect on the trait of interest for specific genotypes.

\section{Genotype effects}

Individual $P_{\mathrm{st}}^{\mathrm{gen}}$ values that provide a quantitative assessment of the genotype contribution to variation at individual temperatures showed highly variable patterns among the different traits and phenotype classes (Additional file 16a). Regardless of the individual temperature, mean genotype effects on developmental timing throughout the vegetative phase were generally very low (Fig. 4a), supporting the results from the analysis of $\mathrm{Q}_{10}$ values (Fig. 3). However, genotype effects on later stages of adult vegetative development seem to increase with higher temperatures (Additional file 16a), which may be the significant effect observed in the ANOVA-based GxE interaction assessment.

Similarly, strong genotype effects at higher temperatures were also observed for reproductive traits. Here, 

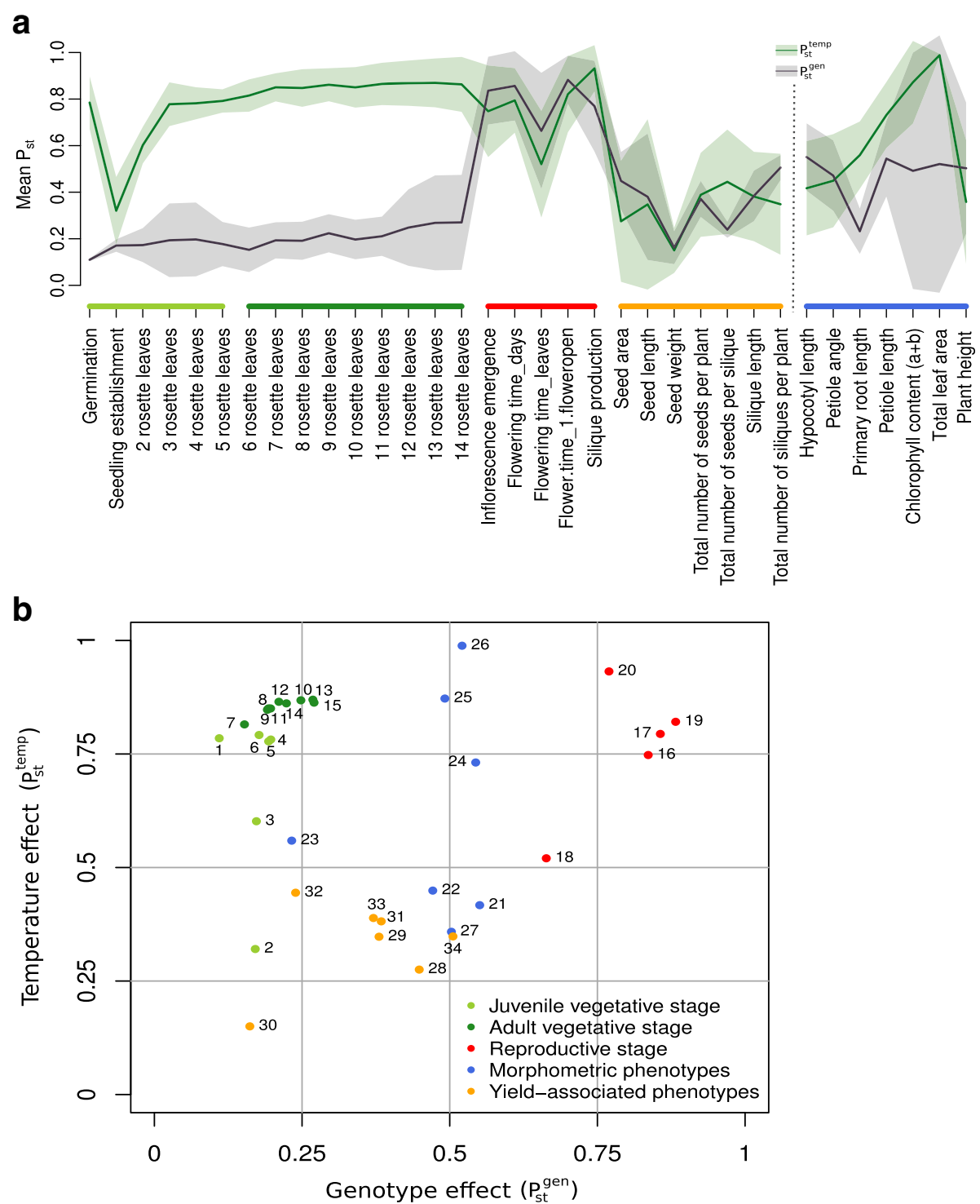

Fig. 4 Genotype and temperature effects on phenotypic variation. a Genotype $\left(P_{\mathrm{st}}^{\text {gen }}\right.$, black $)$ and temperature $\left(P_{\mathrm{st}}^{\text {temp }}\right.$, green) contribution to variation. Solid lines show mean $P_{s t}$ values and shaded areas indicate standard deviations. $\mathbf{b}$ Scatter plot of mean $P_{\mathrm{st}}^{\text {gen }}$ and $P_{\mathrm{st}}^{\text {temp }}$ values over all temperatures and accessions, respectively. Phenotypes are color-coded according to the phenotype classes shown in Fig. 1 and described in Additional file 1. A heatmap of individual $P_{\mathrm{st}}^{\mathrm{gen}}$ and $P_{\mathrm{st}}^{\mathrm{temp}}$ values and a scatter plot including standard deviations are shown in Additional file 16

$P_{\mathrm{st}}^{\mathrm{gen}}$ values at $16{ }^{\circ} \mathrm{C}$ were already considerably higher than for vegetative growth stages and increased further with elevated temperatures (Additional file 16a). A contrasting pattern of decreasing genotype effects with an increase in temperatures was observed for plant height indicating that here, natural variation in growth is higher at lower temperatures. Yield-associated phenotypes in general showed only low genotype effects on variation, indicating that under our experimental conditions variation in trait expression in this category is primarily affected by temperature (Fig. 4a).
Other phenotypes display rather differential or less gradual genotype effects among different temperatures. For example, the genotype impact on variation in hypocotyl and petiole length increases only at $28^{\circ} \mathrm{C}$ whereas the overall genotype contribution to variation from 16 to $24{ }^{\circ} \mathrm{C}$ remains rather low (Additional file 16a). Such patterns of genotype effects may indicate a certain buffering capacity or a threshold for natural variation effects.

In some cases, such as flowering time, a strong genotype effect seems to correlate also with a strong general temperature sensitivity as indicated by the high between- 
accessions variability in $\mathrm{Q}_{10}$ values (Figs. $4 \mathrm{a}$ and $3 \mathrm{~b}$ ). However, this does not seem to be a general principle. In case of root length, for example, low genotype effects were observed (Fig. 4a, b), even though the phenotype in principle was highly sensitive to a change in ambient temperature (Fig. 3b).

\section{Temperature effects}

We also used the variance partitioning approach to analyze the extent of the significant impact of temperature on phenotypic variation that was detected in the $\mathrm{GxE}$ interaction analysis (Additional file 15). Therefore, we calculated the index for temperature effects $\left(P_{\mathrm{st}}^{\text {temp }}\right)$ on the variation of phenotypic plasticity across all four temperatures within each of the ten accessions (Additional file 16b). In contrast to $P_{\mathrm{st}}^{\mathrm{gen}}$, the $P_{\mathrm{st}}^{\mathrm{temp}}$ thus provides information primarily on the temperature-induced variability for each accession individually.

The heatmap representation of temperature effects (Additional file 16b) partially complements the genotype effect results. For example, variation in the timing of vegetative development was highly affected by temperature (high $P_{\mathrm{st}}^{\text {temp }}$ ), whereas $P_{\mathrm{st}}^{\text {gen }}$ values were generally low (Fig. 4a, Additional file 16a, b). Interestingly, temperature effects in juvenile vegetative stages seemed to be lower (for seedling establishment and 2 rosette leave stage) than in later vegetative stages with the exception of germination which showed strong temperature effects in most accessions.

Many traits exhibit highly differential temperature effects among accessions in the sense of one accession demonstrating a particularly strong temperature effect on a specific trait, while another accession may show low to no temperature effects (e.g. chlorophyll content in Ler-1 vs. Bay-0). This is particularly obvious for yield-related traits such as total number of seeds per plant and silique as well as silique length. Here, temperature effects on phenotype variation were low for Col-0, C24 and Bay-0, whereas considerably higher $P_{\mathrm{st}}^{\text {temp }}$ values were determined for the other accessions (Additional file 16b). Accessions which exhibit differential temperature effects on phenotypic variation may be interesting candidates for forward genetic approaches to identify the contributing molecular regulatory components.

\section{Comparison of temperature and genotype effects}

As each phenotypic trait has been assigned a value for genotype and temperature effects, they can easily be compared to assess which of the two has a stronger influence on the phenotypic plasticity. To allow a direct comparison of effects, we compared mean values for
$P_{\mathrm{st}}^{\mathrm{gen}}$ across all temperatures and $P_{\mathrm{st}}^{\mathrm{temp}}$ across all accessions (Fig. 4a, b).

Temperature effects on vegetative development showed a high, largely robust impact with little variance in $P_{\mathrm{st}}^{\mathrm{temp}}$ values, whereas genotype effects were generally low with diverging variances. Genotype effects peak at the transition to the reproductive phase and in some morphometric phenotypes. In general, morphometric parameters show high temperature and varying genotype effects. Phenotypes associated with late developmental stages were generally less affected by both factors indicating an overall buffering effect. Yet, variances in temperature effects tended to be high here, which may indicate genotype-specific thresholds for temperature effects (Fig. 4a, Additional file 16c). A scatter plot representation of mean $P_{\mathrm{st}}^{\mathrm{gen}}$ and $P_{\mathrm{st}}^{\mathrm{temp}}$ values for each trait allows further comparison of phenotypes according to the impact of both factors (Fig. 4b). While vegetative and reproductive phenotypes form tight clusters, morphometric phenotypes displayed a heterogenous pattern. In these traits, temperature responses seem to be affected by natural variation and may thus serve as candidate phenotypes for classic or quantitative forward genetic analyses.

Several yield-associated phenotypes such as total number of seeds, seed size, and seed weight showed varying degrees of temperature sensitivity, likely caused by the partially distinct temperature effects on individual accessions (Fig. 2b, Additional file 17).

The fundamental impact of temperature on the phenotypic responses is also reflected in the results of the principle component analysis (PCA). The PCA was performed on mean-centered and scaled data in order to allow integration of data with different scaling. PC1 which covered $50 \%$ of the observed variation, allowed a clear separation of samples via temperature (Fig. 5a). Here, the differentiation between 16 and $20{ }^{\circ} \mathrm{C}$ seems to be higher than the temperature changes from 20 to $24{ }^{\circ} \mathrm{C}$ and 24 to $28{ }^{\circ} \mathrm{C}$. PC2 explained $\sim 16 \%$ of the variation and separated samples rather by genotype. Here, Rrs-7 and Got-7 showed a clear divergence from other genotypes. Again, this separation is already clear between the temperatures 16 and $20{ }^{\circ} \mathrm{C}$ whereas a further increase in temperature contributed little more to the separation.

\section{Correlation of phenotypic temperature responses}

Finally, we analyzed putative correlations in temperature responses among different phenotypes to assess whether individual phenotype responses are indicative of temperature responses in general. As redundancies of individual phenotypes may bias the analyses several traits were combined in groups for further analyses (e.g. rosette development or flowering traits). We used the 


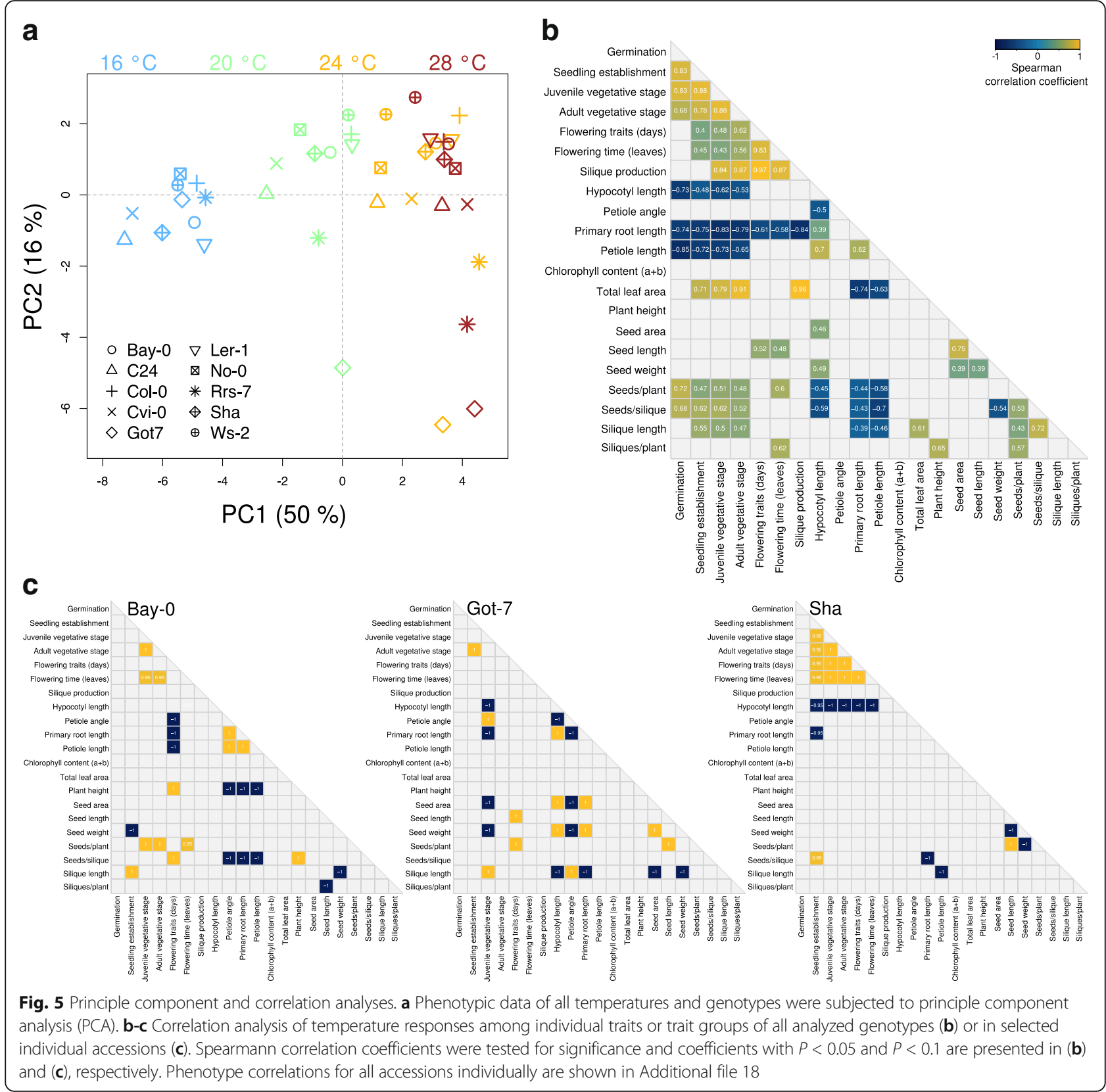

rank-based Spearman correlation coefficients for pairwise comparisons of averaged trait (group) values among all accessions to account for potential non-linear relationships and minimize outlier effects. As to be expected from the varying degrees of genotype and temperature effects on different traits, phenotypic correlations also varied considerably. To filter for robust correlations, only significant correlations $(P<0.05)$ were retained in the analysis (Fig. 5b).

High correlations were detected among traits within the vegetative stage of development (e.g. juvenile and adult vegetative stage), and among traits within the reproductive phase (e.g. flowering traits and the onset of silique production). In addition, temperature-induced reduction in total leaf area correlated strongly with the decrease in developmental time in vegetative and reproductive phases. Similarly, the reduction in developmental times and total leaf area were moderately correlated to the effect on several seed-associated traits (Fig. 5b).

Model temperature phenotypes such as petiole and hypocotyl length showed a positive correlation and were in turn correlated or inversely correlated with several other phenotypes or trait groups. However, temperature responses in primary root length under these experimental conditions showed an even more robust connection to many other traits. Mostly, these 
were inverse correlations with the exception of other seedling traits which were positively correlated with primary root lengths (Fig. 5b).

Due to the differential genotype effects on variation we also wondered whether individual genotypes may show different correlation patterns among phenotypic temperature responses. Calculation of Spearmann correlation coefficients for each individual accession is based on a maximum of four data points per phenotype or trait group which generally results in weaker interactions among samples. Thus, the $P$-value threshold was set to 0.1 in the analysis which retained only the strongest (inverse) correlations. Inspection of the correlation patterns reveals remarkable differences among accessions (Fig. 5c, Additional file 18). For instance, petiole lentgh, angle and primary root length in Bay-0 were all inversely correlated with flowering time, plant height and the number of seeds/silique, whereas in Sha, only hypocotyl lengths showed an inverse correlation with developmental timing in vegetative and reproductive stages. Got-7 even showed unique correlation patterns among early growth responses with inverse correlations among petiole angles and hypocotyl and root lengths, respectively (Fig. 5c). Accession-specific correlations among individual phenotypes should be treated cautiously as these correlations are based on far fewer data points than in the general analysis (Fig. 5b). Nevertheless, the diversity in correlation patterns may indicate differential capacities for temperature responses that result in differential activation or buffering and, thus, in different extents of physiological temperature impacts. On a molecular level this may involve different genetic factors or background-specific epistatic interactions. Elucidation of the underlying mechanisms of differential temperature responses and adaptations may provide essential tools for the modulation of crop responses to elevated ambient temperatures.

\section{Discussion}

Increased ambient temperatures have previously been shown to affect thermomorphogenesis for selected "model" phenotypes. A systematic assessment of developmental and phenotypic plasticity across a complete life cycle has, to the best of our knowledge, been lacking so far. This study aims to provide such a solid base of temperature effects on plants by consecutive profiling of plant growth and development throughout a life cycle of A. thaliana grown in four different ambient temperatures. Furthermore, including several distinct $A$. thaliana accessions reduced potential genotype-specific biases in the data and allowed the analysis of temperature and genotype effects on the variation observed in different phenotypic traits.
All of the 34 analyzed phenotypes were significantly affected by different growth temperatures, natural variation, and GxE interactions, illustrating the fundamental impact of ambient temperature on plant development and the high variability in responses among genotypes (Additional files 4, 5, 6, 7, 8, 9, 10, $11,12,13$ and 15). The variance partitioning approach allowed the further dissection of phenotypes based on the extent of temperature and genotype effects. First, we identified phenotypes that were primarily affected by temperature and showed small genotype-induced variation. Second, we identified phenotypes that additionally or even predominantly showed genotype effects on the observed phenotypic variation.

Developmental timing of juvenile and adult vegetative growth was significantly affected by genotype and temperature (Additional file 15). Yet, temperature was the dominant factor in the observed variation (Figs. 4a, 5a, Additional file 16). Genotype effects on the variation observed during vegetative development, albeit significant, were limited (Fig. 4a). Accordingly, similar accelerations by increasing temperatures were observed in all analyzed genotypes (Additional files 4, 5, $6,7,8,9,10,11,12$ and 13). This observation may be indicative for extensive thermodynamic effects on (conserved) regulatory mechanisms involved in this process. Indeed, thermomorphogenic responses are often speculated to be primarily caused by broad or general effects of free energy changes on biochemical reactions (e.g. enzyme activities). The validity of the early proposed temperature coefficient $\left(\mathrm{Q}_{10}\right)$ for plant development was demonstrated for germination rates and plant respiration [44, 45]. The strong temperature effect on the acceleration of developmental timing throughout the vegetative phase, which was only weakly affected by genotypes supports this theory. When adopting the terms of "passive" and "active" temperature effects as proposed by [46], timing of vegetative development would represent a passive temperature response that might be caused by thermodynamic effects on metabolic rates and enzyme activities or on highly conserved signaling/response components.

On the other hand, phenotypes that show a high degree of genotype and temperature effects might rather be influenced by one or more specific genes that contribute to trait expression in a quantitative manner. As such, these phenotypes would represent "active" temperature effects [46]. However, the involvement of specific signaling elements does not necessarily exclude influences via thermodynamics. In fact, the recently described thermosensing via phyB acts via the promotion of phyB $\mathrm{P}_{\mathrm{FR}}$ to $\mathrm{P}_{\mathrm{R}}$ conversion in a temperature-promoted manner [19, 20]. Natural 
variation in thermomorphogenic responses could be caused by polymorphisms in signaling or response genes ranging from alteration in gene sequence to expression level polymorphism $[47,48]$. As they may provide keys to altered temperature responses that could be utilized in specific breeding approaches, identification of such genes would be of high interest.

In fact, natural allelic variation in the circadian clock components ELF3 and in the regulation of GIGANTEA have recently been shown to directly affect PIF4mediated hypocotyl elongation in response to elevated temperatures [12, 13, 49]. Therefore, PIF4 and PIF4regulating components could be important targets of adaptation to growth in higher ambient temperatures. PIF4 and ELF3 have been shown to be involved in both, temperature-induced hypocotyl elongation and the induction of flowering [12, 13, 21, 50]. However, a lack of general correlation among seedling growth and flowering time responses may indicate that these processes are not universally regulated via the same components. Alternatively, the impact of these signaling components on diverse phenotypes may be more prominent for specific alleles which may be reflected by the diversity in correlation patterns among individual accessions (Fig. 5c, Additional file 18).

Partially, the intraspecific diversity in phenotypic changes in response to elevated ambient temperatures argue against a general explanation of morphological and developmental changes due to passive thermodynamic effects.

Exploiting natural genetic variation to identify genes that are involved in the regulation of temperature effects on specific traits can provide new leads for plant breeding. Particularly, identification of molecular determinants of temperature responses in yield-associated traits should be considered in future studies. The work presented here may inspire new approaches for temperature research in non-reference accessions as some temperature responses were much more pronounced in accessions other than Col-0 (Fig. 3b). Specific approaches will depend on the individual focus of the study which may encompass development, yield- or biomass-associated traits. Each of these aspects is fundamental from an agricultural point of view but molecular factors involved in their temperature responses may differ considerably.

\section{Conclusion}

In conclusion, our work provides a map that allows the dissection of thermomorphogenesis in phenotypic traits that are either robustly affected by temperature or traits that are differentially affected by temperature among different accessions. While robust temperature-sensitive phenotypes might indeed be caused by thermodynamic acceleration of metabolism or highly conserved signaling events, natural genetic variation of temperature responses implicate the relevance of specific regulatory cascades that can be instrumental to future breeding approaches.

\section{Additional files}

Additional file 1: Table of recorded phenotypes and association to phenotype classes. (PDF $12071 \mathrm{~kb}$ )

Additional file 2: Sample sizes, identity and geographic origin of analyzed $A$. thaliana accessions. (PDF 12069 kb)

Additional file 3: Reaction norm plots of each phenotype for each of the analyzed genotypes. (PDF $12067 \mathrm{~kb}$ )

Additional file 4: Summary of Col-0 thermomorphogenesis. (PDF $12069 \mathrm{~kb}$ )

Additional file 5: Summary of Bay-0 thermomorphogenesis. (PDF $12069 \mathrm{~kb}$ )

Additional file 6: Summary of C24 thermomorphogenesis. (PDF $12069 \mathrm{~kb}$ )

Additional file 7: Summary of Cvi-0 thermomorphogenesis. (PDF $12069 \mathrm{~kb}$ )

Additional file 8: Summary of Got-7 thermomorphogenesis. (PDF $12069 \mathrm{~kb}$ )

Additional file 9: Summary of Ler-1 thermomorphogenesis. (PDF $12069 \mathrm{~kb}$ )

Additional file 10: Summary of No-0 thermomorphogenesis. (PDF $12069 \mathrm{~kb}$ )

Additional file 11: Summary of Rrs-7 thermomorphogenesis. (PDF $12069 \mathrm{~kb}$ )

Additional file 12: Summary of Sha thermomorphogenesis. (PDF 12069 kb)

Additional file 13: Summary of Ws-2 thermomorphogenesis.

(PDF $12069 \mathrm{~kb}$ )

Additional file 14: Natural variation in developmental timing. (leaves vs. days). (PDF $12069 \mathrm{~kb}$ )

Additional file 15: GxE interaction analysis results. (PDF $12069 \mathrm{~kb}$ )

Additional file 16: Detailed information on genotype and temperature effects on phenotypic variation. (PDF 12069 kb)

Additional file 17: Temperature effect on yield. (PDF 12069 kb)

Additional file 18: Correlations among temperature responses in individual accessions. (PDF $12069 \mathrm{~kb}$ )

Acknowledgements

We are grateful to Ivo Grosse for critical discussion in the initial phase of this project.

\section{Funding}

This study was supported by the Leibniz Association and a grant from the Deutsche Forschungsgemeinschaft to M.Q. (Qu 141/3-1). Y.P. s supported by the German Centre for Integrative Biodiversity Research (iDiv) Halle-Jena-Leipzig, funded by the Deutsche Forschungsgemeinschaft (FZT 118).

Availability of data and materials

The datasets analysed during the current study are available from the corresponding author on request.

\section{Authors' contributions}

$\mathrm{Cl}, \mathrm{MQ}$, and $\mathrm{CD}$ designed the research and experimental setup. $\mathrm{Cl}, \mathrm{TP}, \mathrm{JB}$ and $\mathrm{KD}$ performed the phenotypic analyses and data collection. Cl, YP and CD analyzed the data. YP, AG-D, and CD designed and performed statistical analyses. $\mathrm{Cl}, \mathrm{YP}, \mathrm{MQ}$, and $\mathrm{CD}$ interpreted data, prepared figures and wrote the manuscript.

Ethics approval and consent to participate

Not applicable.

Consent for publication

Not applicable.

Competing interests

The authors declare that they have no competing interests. 


\section{Publisher's Note}

Springer Nature remains neutral with regard to jurisdictional claims in published maps and institutional affiliations.

\section{Author details \\ Institute of Agricultural and Nutritional Sciences, Martin Luther University Halle-Wittenberg, Betty-Heimann-Str. 5, 06120 Halle (Saale), Germany. ${ }^{2}$ Department of Molecular Signal Processing, Leibniz Institute of Plant Biochemistry, Weinberg 3, 06120 Halle (Saale), Germany. ${ }^{3}$ German Centre for Integrative Biodiversity Research (iDiv) Halle-Jena-Leipzig, Deutscher Platz 5e, 04103 Leipzig, Germany. ${ }^{4}$ Institute of Computer Science, Martin Luther University Halle-Wittenberg, Von-Seckendorff-Platz 1, 06099 Halle (Saale), Germany.}

Received: 13 March 2017 Accepted: 25 June 2017

Published online: 06 July 2017

\section{References}

1. Quint M, Delker C, Franklin KA, Wigge PA, Halliday K, van Zanten M. Molecular and genetic control of plant thermomorphogenesis. Nat Plants. 2016;2:15190.

2. Peng S, Huang J, Sheehy JE, Laza RC, Visperas RM, Zhong X, et al. Rice yields decline with higher night temperature from global warming. Proc Natl Acad Sci. 2004;101:9971-5.

3. Moore FC, Lobell DB. The fingerprint of climate trends on European crop yields. Proc Natl Acad Sci U S A. 2015;112:2670-5.

4. IPCC. Climate change 2013: The physical science basis. Fifth assessment report. [Internet]. UNEP/WMO; Available from: http://www.ipcc.ch/ report/ar5/wg1/.

5. Lobell DB, Gourdji SM. The influence of climate change on global crop productivity. Plant Physiol. 2012;160:1686-97.

6. Fitter AH, Fitter RSR. Rapid changes in flowering time in british plants. Science. 2002;296:1689-91.

7. CaraDonna PJ, ller AM, Inouye DW. Shifts in flowering phenology reshape a subalpine plant community. Proc Natl Acad Sci. 2014;111:4916-21.

8. Thuiller W, Lavorel S, Araújo MB, Sykes MT, Prentice IC. Climate change threats to plant diversity in Europe. Proc Natl Acad Sci. 2005;102:8245-50.

9. Gray WM, Östin A, Sandberg G, Romano CP, Estelle M. High temperature promotes auxin-mediated hypocotyl elongation in Arabidopsis. Proc Natl Acad Sci. 1998;95:7197-202.

10. Zanten M van, Voesenek LACJ, Peeters AJM, Millenaar FF. Hormone- and light-mediated regulation of heat-induced differential petiole growth in Arabidopsis. Plant Physiol 2009:151:1446-1458.

11. Balasubramanian S, Sureshkumar S, Lempe J, Weigel D. potent induction of Arabidopsis thaliana flowering by elevated growth temperature. PLoS Genet. 2006;2:e106

12. Raschke A, Ibañez C, Ullrich KK, Anwer MU, Becker S, Glöckner A, et al. Natural variants of ELF3 affect thermomorphogenesis by transcriptionally modulating PIF4-dependent Auxin responses. BMC Plant Biol. 2015:15:197.

13. Box MS, Huang BE, Domijan M, Jaeger KE, Khattak AK, Yoo SJ, et al. ELF3 controls thermoresponsive growth in Arabidopsis. Curr Biol. 2015;25:194-9.

14. Zhu W, Ausin I, Seleznev A, Méndez-Vigo B, Picó FX, Sureshkumar S, et al. Natural variation identifies ICARUS1, a Universal Gene Required for Cell Proliferation and Growth at High Temperatures in Arabidopsis thaliana. PLoS Genet. 2015;11:e1005085.

15. Lutz U, Posé D, Pfeifer $M$, Gundlach $H$, Hagmann J, Wang C, et al. Modulation of ambient temperature-dependent flowering in Arabidopsis thaliana by natural variation of FLOWERING LOCUS M. PLoS Genet. 2015;11:e1005588.

16. Sanchez-Bermejo E, Balasubramanian S. Natural variation involving deletion alleles of FRIGIDA modulate temperature-sensitive flowering responses in Arabidopsis thaliana. Plant Cell Environ. 2016;39:1353-65.

17. Ma D, Li X, Guo Y, Chu J, Fang S, Yan C, et al. Cryptochrome 1 interacts with PIF4 to regulate high temperature-mediated hypocotyl elongation in response to blue light. Proc Natl Acad Sci. 2016;113:224-9.

18. Sanchez-Bermejo E, Zhu W, Tasset C, Eimer H, Sureshkumar S, Singh R, et al. Genetic architecture of natural variation in thermal responses of Arabidopsis. Plant Physiol. 2015;169:647-59.

19. Jung J-H, Domijan M, Klose C, Biswas S, Ezer D, Gao M, et al. Phytochromes function as thermosensors in Arabidopsis. Science. 2016:354:886-9.

20. Legris M, Klose C, Burgie ES, Costigliolo C, Neme M, Hiltbrunner A, et al. Phytochrome $B$ integrates light and temperature signals in Arabidopsis. Science. 2016;354:897-900.
21. Koini MA, Alvey L, Allen T, Tilley CA, Harberd NP, Whitelam GC, et al. High temperature-mediated adaptations in plant architecture require the bHLH transcription factor PIF4. Curr Biol. 2009;19:408-13.

22. Franklin KA, Lee SH, Patel D, Kumar SV, Spartz AK, Gu C, et al. Phytochromeinteracting factor 4 (PIF4) regulates auxin biosynthesis at high temperature. Proc Natl Acad Sci U S A. 2011;108:20231-5.

23. Proveniers MCG, van Zanten M. High temperature acclimation through PIF4 signaling. Trends Plant Sci. 2013;18:59-64.

24. Toledo-Ortiz G, Johansson H, Lee KP, Bou-Torrent J, Stewart K, Steel G, et al. The HY5-PIF regulatory module coordinates light and temperature control of photosynthetic gene transcription. PLoS Genet. 2014;10: e1004416.

25. Delker C, Sonntag L, James GV, Janitza P, Ibañez C, Ziermann H, et al. The DET1-COP1-HY5 Pathway Constitutes a Multipurpose Signaling Module Regulating Plant Photomorphogenesis and Thermomorphogenesis. Cell Rep. 2014;9:1983-9.

26. Gangappa SN, Kumar SV. DET1 and HY5 Control PIF4-Mediated Thermosensory Elongation Growth through Distinct Mechanisms. Cell Rep. 2017:18:344-51.

27. McKhann HI, Camilleri C, Bérard A, Bataillon T, David JL, Reboud X, et al. Nested core collections maximizing genetic diversity in Arabidopsis thaliana. Plant J. 2004;38:193-202

28. Delker C, Pöschl Y, Raschke A, Ullrich K, Ettingshausen S, Hauptmann V, et al Natural variation of transcriptional auxin response networks in Arabidopsis thaliana. Plant Cell. 2010;22:2184-200.

29. Scholl RL, May ST, Ware DH. Seed and molecular resources for arabidopsis. Plant Physiol. 2000;124:1477-80.

30. Lincoln C, Britton J, Estelle M. Growth and development of the axr1 mutants of Arabidopsis. Plant Cell. 1990;2:1071-80.

31. ImageJ: http://imagej.nih.gov/ij/

32. RootDetection: http://www.labutils.de/rd.html

33. Boyes DC, Zayed AM, Ascenzi R, McCaskill AJ, Hoffman NE, Davis KR, et al. Growth stage-based phenotypic analysis of Arabidopsis: a model for high throughput functional genomics in plants. Plant Cell. 2001;13: 1499-510.

34. Porra RJ, Thompson WA, Kriedemann PE. Determination of accurate extinction coefficients and simultaneous equations for assaying chlorophylls $\mathrm{a}$ and $\mathrm{b}$ extracted with four different solvents: verification of the concentration of chlorophyll standards by atomic absorption spectroscopy. Biochim Biophys Acta BBA - Bioenerg. 1989;975:384-94.

35. R Core Team. R: a language and environment for statistical computing [Internet]. Vienna: R Foundation for Statistical Computing; 2015. https:// www.R-project.org

36. Nicotra AB, Atkin OK, Bonser SP, Davidson AM, Finnegan EJ, Mathesius U, et al. Plant phenotypic plasticity in a changing climate. Trends Plant Sci. 2010; 15:684-92.

37. Whitman D, Agrawal A. What is phenotypic plasticity and why is it important? Phenotypic Plast. Insects. 2009: http://dx.doi.org/10.1201/b10201-2

38. Loveys BR, Atkinson LJ, Sherlock DJ, Roberts RL, Fitter AH, Atkin OK. Thermal acclimation of leaf and root respiration: an investigation comparing inherently fast- and slow-growing plant species. Glob Change Biol. 2003;9: 895-910.

39. Storz JF. Contrasting patterns of divergence in quantitative traits and neutral DNA markers: analysis of clinal variation. Mol Ecol. 2002;11:2537-51.

40. Leinonen T, Cano JM, Mäkinen H, Merilä J. Contrasting patterns of body shape and neutral genetic divergence in marine and lake populations of threespine sticklebacks. J Evol Biol. 2006:19:1803-12.

41. NASC/ABRC. https://www.arabidopsis.org/abrc/catalog/natural_accession_9.html

42. Gay L, Neubauer G, Zagalska-Neubauer M, Pons J-M, Bell DA, Crochet P-A. Speciation with gene flow in the large white-headed gulls: does selection counterbalance introgression? Heredity. 2008;102:133-46.

43. Whitlock MC. Evolutionary inference from QST. Mol Ecol. 2008;17:1885-96.

44. Hegarty TW. temperature coefficient (q10), seed germination and other biological processes. Nature. 1973;243:305-6.

45. Atkin OK, Tjoelker MG. Thermal acclimation and the dynamic response of plant respiration to temperature. Trends Plant Sci. 2003:8:343-51.

46. Penfield S, MacGregor D. Temperature sensing in plants. In: Franklin Ka, Wigge P a, editors. Temperature and Plant Development. Hoboken: John Wiley \& Sons, Inc; 2014. p. 1-18.

47. Delker C, Quint M. Expression level polymorphisms: heritable traits shaping natural variation. Trends Plant Sci. 2011;16:481-8. 
48. Alonso-Blanco C, Aarts MGM, Bentsink L, Keurentjes JJB, Reymond M, Vreugdenhil $D$, et al. What has natural variation taught us about plant development, physiology, and adaptation? Plant Cell. 2009;21:1877-96.

49. de Montaigu A, Giakountis A, Rubin M, Tóth R, Cremer F, Sokolova V, et al. Natural diversity in daily rhythms of gene expression contributes to phenotypic variation. Proc Natl Acad Sci. 2015;112:905-10.

50. Kumar SV, Lucyshyn D, Jaeger KE, Alós E, Alvey E, Harberd NP, et al. Transcription factor PIF4 controls the thermosensory activation of flowering. Nature. 2012;484:242-5.

Submit your next manuscript to BioMed Central and we will help you at every step:

- We accept pre-submission inquiries

- Our selector tool helps you to find the most relevant journal

- We provide round the clock customer support

- Convenient online submission

- Thorough peer review

- Inclusion in PubMed and all major indexing services

- Maximum visibility for your research

Submit your manuscript at www.biomedcentral.com/submit 